\title{
Age Difference in Perceived Ease of Use, Curiosity, and Implicit Negative Attitude toward Robots
}

\author{
SUNG-EN CHIEN, National Taiwan University \\ LI CHU, National Taiwan University and The Chinese University of Hong Kung \\ HSING-HAO LEE, CHIEN-CHUN YANG, FO-HUI LIN, and PEI-LING YANG, \\ National Taiwan University \\ TE-MEI WANG, Industrial Technology Research Institute \\ SU-LING YEH, National Taiwan University
}

\begin{abstract}
Understanding older adults' attitudes toward robots has become increasingly important as robots have been introduced in various settings, such as retirement homes. We investigated whether there are age differences in both implicit and explicit attitudes toward robots after interacting with an assistive robot. Twenty-four younger and 24 older adults were recruited. Explicit attitudes were measured by self-reported questionnaires both before and after interacting with the robot. State curiosity toward robots was also measured as a momentary form of explicit attitude. Implicit attitude was measured via an implicit association test. Our results showed that (1) both older and younger adults had more positive explicit attitudes toward robots after interaction; (2) older adults had lower state curiosity than younger adults, however, their state curiosity would be up to the same level as younger adults when they perceived the robot with higher levels of personal association; and (3) the implicit association between robots and negative words was stronger for older adults than younger adults, suggesting that older adults had more implicit negative attitude toward robots. The results suggest that, despite older adults' relatively more negative implicit attitude toward robots, personally relevant positive experiences could help improve their explicit attitudes toward robots.
\end{abstract}

\section{CCS Concepts: • Applied computing $\rightarrow$ Law, social and behavioral sciences; Psychology;}

Additional Key Words and Phrases: Implicit attitude, explicit attitude, curiosity, age differences

\section{ACM Reference format:}

Sung-En Chien, Li Chu, Hsing-Hao Lee, Chien-Chun Yang, Fo-Hui Lin, Pei-Ling Yang, Te-Mei Wang, and Su-Ling Yeh. 2019. Age Difference in Perceived Ease of Use, Curiosity, and Implicit Negative Attitude toward Robots. ACM Trans. Hum.-Robot Interact. 8, 2, Article 9 (May 2019), 19 pages.

https://doi.org/10.1145/3311788

\footnotetext{
This project is supported by the Industrial Technology Research Institute (ITRI) of Taiwan.

Authors' addresses: S.-E. Chien, National Taiwan University, No. 1, Sec. 4, Roosevelt Rd., Department of Psychology, Taipei, Taiwan, 10617; email: chiensungen@gmail.com; L. Chu, Department of Psychology, The Chinese University of Hong Kong, Room 345, Sino Building, Shatin, New Territories, Hong Kong SAR, China; email: lichu@link.cuhk.edu.hk; H.-H. Lee, C.-C. Yang, F.-H. Lin, and P.-L. Yang, National Taiwan University, No. 1, Sec. 4, Roosevelt Rd., Department of Psychology, Taipei 10617, Taiwan; emails: hsinghaolee@gmail.com, \{b02207001, b04207026\}@ntu.edu.tw, plyang2@illinois.edu; T.-M. Wang, Industrial Technology Research Institute Chutung, Electronic and Optoelectronic System Research Laboratories, 195, Sec. 4, Chung Hsing Rd., Hsinchu, Taiwan, 31040; email: TeMeiWang@itri.org.tw; S.-L. Yeh, National Taiwan University; email: suling@g.ntu.edu.tw.

Permission to make digital or hard copies of all or part of this work for personal or classroom use is granted without fee provided that copies are not made or distributed for profit or commercial advantage and that copies bear this notice and the full citation on the first page. Copyrights for components of this work owned by others than the author(s) must be honored. Abstracting with credit is permitted. To copy otherwise, or republish, to post on servers or to redistribute to lists, requires prior specific permission and/or a fee. Request permissions from permissions@acm.org.

(C) 2019 Copyright held by the owner/author(s). Publication rights licensed to ACM. 2573-9522/2019/05-ART9
}

https://doi.org/10.1145/3311788 


\section{INTRODUCTION}

Population aging is a global trend and the need for robotics technology for assistance has become increasingly necessary in many countries. Indeed, population aging has become obvious in many parts of the world, especially in Asia. For example, Taiwan became an aging society (more than $7 \%$ of the population was 65 years old or older) in 1993 and has turned into an aged society (13.9\% of Taiwan's population is currently above age 65 [1]) in 25 years. With the rapid growth of the aging population, labor shortage in caregiving for older adults is expected [1]. Fortunately, advances in robotics technology have offered various novel products that have the potential to provide personal assistance and facilitation of social interaction for older adults. With these new inventions, researchers have become interested in exploring the applicability of introducing robotic products to older adults [2].

However, it is unclear whether older adults are willing to accept such robotic products for assistance. According to a model on older adults' technology acceptance [3], having intention to use the targeted technology is a prerequisite for product exploration and eventual product adoption/ rejection. As the key component of user intention is users' attitudes toward the product, it is imperative to understand older adults' attitudes toward robots, and whether it would be different from younger adults.

Attitudes can be either explicit or implicit, and each has its own manifestation in human behavior. Explicit attitude is typically measured using a self-reported questionnaire, which captures a person's controllable, deliberate, and conscious perceptions or judgements. Conversely, implicit attitude usually functions without one's full awareness or control but can be tested using the Implicit Association Task [4]. Interestingly, these two types of attitudes may or may not be closely associated with each other. Based on a literature review, correlations between implicit and explicit attitudes varied greatly from weakly positive (below .20) to strongly positive (above .75), depending on the group of interest and the reference group [5]. Moreover, the manifestations of implicit and explicit attitudes in behavior can be quite different. Previous studies on explicit and implicit prejudice toward African or White Americans demonstrated that explicit attitudes were more predictive of deliberative behaviors (e.g., verbal behaviors); whereas implicit attitudes were more reflective of spontaneous behaviors (e.g., spontaneous laughter) [6]. It appears that both types of attitudes translated into behavioral outcomes but had different manifestations, which highlights the value of studying implicit and explicit attitudes jointly. These two unique constructs provide an opportunity to examine how a particular group (e.g., older adults) view a certain concept or object (e.g., robots). Understanding how older adults' explicit and implicit attitudes toward robots may differ from younger adults provides us with further insight on how to better design, manufacture, and deliver future technologies dedicated for older adults.

Explicit attitudes toward robots are important measures to consider, especially among older adults because previous research demonstrated that older adults' explicit attitudes might affect their acceptance of robots after interaction [7] as well as other technologies such as spreadsheet software [8]. Older adults with more positive attitudes toward robots were more likely to use robots, and these participants who used the robots showed improved attitudes toward robots over time [9]. Perceived enjoyment, ease of use, usefulness, and trust all affected older adults' intention to use a robotic system $[7,10]$. Beer et al. [11] administered pre- and post-exposure questionnaires about older adults' attitudes toward robots. They found that older adults' attitudes became more positive after observing a mobile manipulator robot demonstrating functions such as delivering medication and organizing home objects [11]. Chen et al. [12] investigated older adults' acceptance of a robot for partner dance-based exercise both before and after performing a simple partnered stepping task with it. The results showed that participants tended to perceive the robot as easier 
to use after interacting with it [12]. However, previous studies usually measured older adults' attitudes without comparing with other age groups such as younger adults. Thus, it is still unclear whether there is an age difference in explicit attitudes after successfully interacting with robots.

The first goal of the current study was to compare changes in explicit attitudes toward robots across different age groups after human-robot interaction. It is known that demographic factors such as gender, culture, and education level would affect older adults' acceptance toward robots [13-15]. Bartneck et al. [15] compared participants' acceptance toward robots and found that participants from the United States had more positive attitudes than participants from other countries (e.g., Japan, Mexico). However, they did not find age effect in their study, probably due to around half of their participants $(N=467)$ were recruited through online communities and might have a self-selection bias. We controlled participants' demographic factors between age groups to minimize the effects of these demographic factors. We also asked our participants to interact with the robot with specific tasks (e.g., ask the robot to dance). Experimenters assisted participants in completing these required tasks in 20 minutes to ensure that participants had similar interacting experience and duration.

Studies using questionnaires to measure older adults' explicit attitudes toward robots often reported inconsistent results [16-19]. For example, Nomura et al. [15] showed that older adults did not show stronger negative attitudes toward robots; they even felt more friendliness toward robots than younger adults [17]. Ezer et al. [20] found that older adults were as willing as younger adults to have a robot to help with housework. Yet, Scopelliti et al. [18] showed that older adults were more fearful of having a robot at home compared to other age groups due to lower familiarity with technology. The discrepancy may be explained by individual differences in prior exposure to robots. Various studies $[11,15]$ asked participants to report their attitude toward robots based on their layperson perception. The source of impression formation may explain why some studies found negative while others found positive attitudes toward robots among older adults.

In the present study, instead of getting attitudinal ratings for individuals' general impression of robots or feelings toward a lab-based uncommercialized robot, we utilized a commercialized social robot that was designed specifically for human-robot interaction. Moreover, according to the selective engagement theory [21], personal relevance is an important factor in older adults' decision-making process; that is, older adults tend to favor options that they feel personally connected to or meaningful. Thus, direct interactions may further enhance personal relevance, which has the potential of improving older adults' explicit attitudes toward the object of interest (i.e., robot).

In addition to the traditional measure of explicit attitudes toward technology, state curiosity is a promising subtype of explicit attitude that has not been well-explored. Curiosity can be defined as a person's willingness and desire to obtain information, which can be measured at both trait and state levels [22]. Trait curiosity reflects individuals' overall tendency to seek for novel information and experience (e.g., a person who is always curious versus a person who is seldom curious); state curiosity demonstrates individuals' momentary desire for information (e.g., a person who is curious now toward a particular object versus a person who is not curious toward the same object). In the current study, in addition to measuring one's general tendency to be curious (i.e., trait curiosity) as a control variable, we focused on state curiosity, which allows us to capture individuals' information seeking desire toward a particular object, such as a robot, at a given moment. Such motivational desire is predictive of future initiation of learning, engagement, and acceptance of the robot, which makes curiosity unique from other measures of explicit attitudes. However, previous studies rarely examined age difference in curiosity toward technology. Our second goal in the current study was to investigate age difference in state curiosity toward robots, which could be conceptualized as state manifestation of individual's explicit attitude. 
Preference of robot over human (or vice versa) may be different based on preferred functions. Smarr et al. [23] found that older adults preferred robot assistance over human assistance for chores, object manipulation, and information management (e.g., doing laundry, opening and closing doors/drawers, reminder of appointments) Yet, they preferred human assistance over robot assistance for personal care activities (e.g., taking a bath, shaving, washing/combing hair) Adopting the view that preference for robots may be inconsistent due to functions, it is possible that older adults may, in general, have more negative attitudes toward robots but evaluate robots as good for certain tasks or purposes (e.g., robots for navigation in an exhibition). Rydell and McConnell [24] also showed that explicit attitude could be rapidly shaped by explicit intention while the change in implicit attitude required repeated pairing between attitude toward robots and evaluation over time. Therefore, it is possible that explicit attitude reflects impressions toward robots with specific functions (e.g., robots for navigation), while implicit attitude reflects general attitudes (e.g., positive/negative impression) toward robots regardless of their functions, which might be more stable than explicit attitude. Measuring individuals' implicit attitude could bring more insights unobtainable by explicit measures. It could be helpful to see the big picture of individuals' attitudes toward robots.

Indeed, implicit methods for measuring negative attitude could reveal additional information beyond what is provided by explicit methods. MacDorman et al. [25] investigated whether culture would affect people's implicit attitude toward robots via comparing attitudes toward robots among faculty members of an American and a Japanese university. Despite the failure to find any cultural difference, their results revealed an interesting aspect of the dissociation of explicit and implicit attitudes. Although both American and Japanese participants explicitly rated people as more threatening than robots, implicit measures suggested that they associated weapons with robots more than humans. They also found that older participants (above age 43.9) associated robots with weapons more strongly than younger participants, suggesting that older participants implicitly felt that robots were more threatening than people. Following this, it is thus likely that older adults would associate robots with negative emotion to a greater extent than younger adults. However, MacDorman and colleagues aimed to examine cultural differences in their study based on the hypothesis that the Japanese participants should have more experiences with robots, because robots contributed in many domains (e.g., manufacturing, healthcare, and entertainment) of the Japanese society. Since the primary focus of MacDorman et al. was to examine cultural difference, they did not control for participants' age [25]. Yet, it is possible that age affects implicit attitude toward robots. Hence, the third goal of the present study was to examine whether older adults showed more negative implicit attitude toward robots compared to younger adults.

In summary, the current study had three main goals. First, we aimed to compare the change in explicit attitudes toward robots between younger and older adults as a result of interacting with the robot. We hypothesized that explicit attitudes toward robots would be rapidly improved if participants could successfully interact with the robot, and we expected that older and younger adults would show the same pattern of attitude change. In other words, we expected that there should be a main effect of pretest-posttest difference, but no age difference. Second, we aimed to investigate age difference in state curiosity toward robots after interacting with the robot. According to the selective engagement theory [21], older adults tend to be more selective (i.e., in terms of how they allocate their cognitive resources) based on personal relevance, and evidence has indeed shown that curiosity or information seeking behaviors tend to decline with age [26]. Priniski and colleagues [27] suggested that there are three levels of personal relevance, namely personal association, personal usefulness, and personal identification. Personal association is the perception that something is connected to self in some way; personal usefulness is the perception that something 
can help oneself to achieve one's goal; identification is defined as the incorporation of something in one's self identity. Among the three, personal association, perceiving some links between the object of interest and oneself, was indicated as the easiest to be manipulated. Hence, if older adults become more selective in whether they want to learn a particular technology based on personal association, robot design should manipulate personal association to increase technology acceptance. Taken together, measuring older adults' curiosity could capture their information seeking desire toward robots, which is predictive of acceptance of robots. And this desire could be modulated by personal association: Higher personal association could enhance individual's curiosity toward robots and consequently enhance the acceptance of robots. Accordingly, we predicted that older adults might be less curious about robots than younger adults, and this age difference could be moderated by personal association. Since we were interested in the association between individuals' curiosity and perceived personal association at a given moment instead of the change of curiosity due to direct interaction, we only measured curiosity in the posttest. Third, we aimed to test whether older adults would display more negative implicit attitude toward robots in comparison to their younger counterparts. We hypothesized that older adults would have stronger negative implicit attitude than younger adults, and this age effect would persist over time since implicit attitude is more stable and thus changes slowly. There should be no differences in implicit attitude before and after interacting with the robot; thereby, we measured participants' implicit attitude in the posttest.

To examine our hypotheses, we first measured both older and younger participants' explicit attitudes toward robots before and after interacting with robots via inventories. By doing this, we examined whether direct interaction with robots changed participants' attitudes toward robots or not. Then, we measured curiosity and applied an implicit association test (IAT) in the posttest to examine whether older adults showed a lower level of state curiosity and more negative implicit attitude toward robots compared to younger adults.

\section{PARTICIPANTS AND METHODS}

\subsection{Participants}

This study was approved by the research ethics committee of National Taiwan University (REC code: 201706HS062). Since we aimed to compare attitudes toward robots between younger and older adults, we intentionally recruited participants from different age populations. We recruited college students as the younger participants. For older participants, we followed the United Nations' age cutoff of later adulthood, which starts from age 60 [28]. We recruited people who were at or above 60 years old. One of the older participants was 59 years old when he/she participated in the present study. However, he/she was only a few days away from becoming 60, therefore we still included his/her data in the present study. Twenty-four younger adults $\left(M_{\text {age }}: 21.46\right.$, range $=20$ to 24 years; half male and half female) and 24 older adults $\left(M_{\text {age }}: 66.63\right.$, range $=59$ to 86 years; half male and half female) were recruited from the campus or the neighborhood of National Taiwan University. The detailed demographic information appears in Table 1. All participants were relatively well-educated and healthy (i.e., without neuropsychological disorder) and had normal or corrected-to-normal vision. Gender and education level between two age groups were matched.

\subsection{The Assistive Robot}

We used the Zenbo robot (Figure 1) to interact with our participants. Zenbo is an assistive robot with an anthropomorphic face currently available on the market, designed by AsusTek Computer 
Table 1. Demographic Information of Participants

\begin{tabular}{cccc}
\hline Group $(N)$ & Age range & ${\text { Mean } \text { Age }^{\mathrm{a}}}$ & Years of Education $^{\mathrm{a}}$ \\
\hline Younger adults $(24,12$ females) & $20-24$ & $21.46 \pm 1.44$ & $15.33 \pm 1.34$ \\
Older adults $(24,12$ females $)$ & $59-86$ & $66.63 \pm 5.81$ & $15.67 \pm 3.02$ \\
\hline
\end{tabular}

${ }^{\mathrm{a}}$ Data are presented as mean $\pm \mathrm{SD}$.

Fig. 1. Zenbo Home Robot that shows a shy expression, which was one of the 12 interactions experienced by the participants when they interacted with Zenbo.

Table 2. Task List Specifying the Functions for Participants to Experience

\begin{tabular}{|c|c|c|}
\hline Function & Command & Robot's reaction \\
\hline Wake up the robot & "Hey! Zenbo!” & Wait for commands \\
\hline Open function list & "What can you do?" & Display function list on display \\
\hline Date report & $\begin{array}{l}\text { "What date is tomorrow in lunar } \\
\text { calendar?" }\end{array}$ & Report date in lunar calendar \\
\hline Weather forecast & $\begin{array}{l}\text { "What is the weather in Taipei } \\
\text { today?" }\end{array}$ & Report weather \\
\hline Basic interaction & Stroke its head & Show a shy expression \\
\hline Conversion & $\begin{array}{l}\text { "Tell me the three principles of } \\
\text { robot." }\end{array}$ & $\begin{array}{l}\text { Answer the three principles of } \\
\text { robot }\end{array}$ \\
\hline News & "Any news today?" & Search news on the Internet \\
\hline Remote control & $\begin{array}{l}\text { Remotely control Zenbo via } \\
\text { experimenter's mobile phone }\end{array}$ & $\begin{array}{l}\text { Receive commands from } \\
\text { experimenter's smartphone }\end{array}$ \\
\hline Following & "Follow me" & Follow the participant \\
\hline Dance & "Can you dance?" & Dance \\
\hline Entertainment & "Tell me a story." & Tell a story to the participant \\
\hline Photo & "I want to take a selfie." & Take a picture of the participant \\
\hline
\end{tabular}

Inc. to help people do daily chores such as making calls, searching online information, and reminding someone to take medication on time. Other functions related to education, entertainment, and health care are also embedded. To examine the effect of human-robot interaction, we specified 12 main functions of Zenbo for participants to experience (Table 2). We will address Zenbo as the assistive robot from this point onward for simplicity. 


\subsection{Explicit Attitude Measures}

Several inventories were used in the present study to measure explicit attitudes toward robots, including the Negative Attitudes toward Robots Scale (NARS) [29], the Technology Acceptance Model (TAM) [30, 31] and the Subjective Technology Adaptivity Inventory (STAI) [32]. We also measured participants' state curiosity toward robots [33] as an additional explicit measure of attitude. Trait curiosity [22] was measured in addition as a control variable. We translated these inventories into Chinese for our participants.

NARS was used to measure participants' negative attitudes toward robots [16] and included 11 items with 5-point Likert scales. There were three subscales [34]: future/social influence (FSI, 3 items, score range: 3-15), relational attitudes (RA, 5 items, score range: 5-25), and actual interactions and situations (AIS, 3 items, score range: 3-15). Higher NARS scores represent a stronger negative attitude toward robots. Based on the result of NARS, we could explain how people view and evaluate robots before and after their interaction with the assistive robot (pretest: $\alpha=.78$; posttest: $\alpha=.76$ ).

TAM is a model proposed by Davis [30] that has been widely used to assess people's acceptance toward technology products [30,31]. It includes in total 10 items with a 5-point Likert scale. Since we were interested in what role an assistive robot could play in people's everyday lives, we adopted TAM2 [35] to examine participants' ratings because of its higher validity and reliability. The chosen factors were (1) perceived usefulness (PU, 4 items, score range: 4-20), (2) perceived ease of use (PEU, 4 items, score range: $4-20$ ), and (3) intention to use (IU, 2 items, score range: $2-10$ ). Higher TAM scores represent higher acceptance of technology products (pretest: $\alpha=.90$; posttest: $\alpha=.85$ ).

STAI is a 5-point, 12-item inventory designed to estimate the adaptivity of technology specifically for older adults [32]. It contains three subscales (4 items in each subscale, score range: 4-20) for investigating different domains of adaptivity: (1) perceived adaptivity utility (PAU), (2) technology-related goal-engagement (TGE), and (3) perceived safety of technology (PST). Higher STAI scores represent higher adaptivity of technology. Based on these results, we could understand the behavioral and mental competence when people face new environmental and technological demands (pretest: $\alpha=.83$; posttest: $\alpha=.83$ ).

State curiosity toward robots is a 7-point, 3-item measure with a score range of 3 to 21 [33]. It was originally designed to measure curiosity toward sports in general, but we adjusted the items in order to fit the purpose of this study (i.e., replacing "this sport" with "this robot"). These items included "How curious do you feel about this robot?", "How likely would you be to spend time watching a video that introduces this robot?", and "How much do you want to know about this robot?" The reliability was good $(\alpha=.88)$. Trait curiosity was a 7-point, 7-item measure with a score range of 7 to 49 [22]. The reliability was below standard ( $\alpha=.66$ ), but we kept the measure since it was used as a control variable only. Unique from the other explicit attitudinal measures, state curiosity toward robots offers a more direct way to capture the subtle difference in technology-related intentions between younger and older adults. By controlling for the trait curiosity, we eliminated the effect of age-related curiosity mean differences. Finally, we measured personal association using a 5-point, single item (i.e., "To what extent do you think the robot is similar to you?") in order to address the second goal of this study.

\subsection{Implicit Attitude Measure}

The IAT measures the strength of implicit associations between a concept and attributions (e.g., a category as positive or negative) using participants' reaction times [36]. This measure gives us the opportunity to compare participants' automatic evaluative associations of two target concepts 
(A) Target Concept: Robot

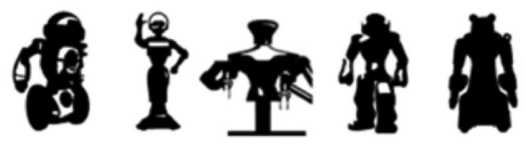

(B) Target Concept: Human

\section{1}

(C) Attribute dimensions of robot impression IAT

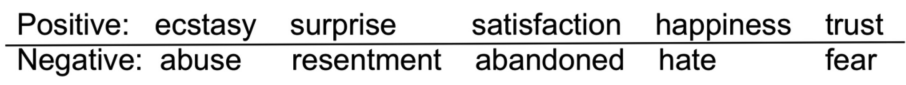

Fig. 2. Images and words used in the IAT test. (A) Silhouettes of humanoid robots to represent the target concept robot. (B) Silhouettes of humans to represent the target concept human. (C) Five positive words for positive impression (ecstasy, surprise, satisfaction, happiness, and trust; upper row) and five negative words for negative impression (abuse, resentment, hate, abandoned, and fear; bottom row) used for the attribute dimension of impression toward robots.

(e.g., robot vs. human) along an attribute dimension (e.g., negative vs. positive emotions) based on participants' reaction time in the combined categorization task: Shorter reaction time represents stronger association between target concept and attribute dimension. It could avoid social desirability since participants are asked to respond as fast as possible in categorization tasks without knowing the purpose of the test.

We adopted the IAT to measure participants' implicit attitude toward robots compared to that toward humans. We hypothesized that older adults would have a more negative attitude toward robots than toward humans. Hence, we used five silhouettes of humanoid robots to represent the target concept "robot" and five silhouettes of humans to represent the target concept "human." The self-made robot and human silhouettes (Figure 2(a) and 2(b)) were made in the same way as in MacDorman et al.'s study, which compared implicit attitude toward robots for participants in the U.S. and Japan [25]. Five positive (ecstasy, surprise, satisfaction, happiness, and trust) and five negative words (abuse, resentment, hate, abandoned, and fear; Figure 2(c)) were used for the attribute dimension of attitude toward robots. These words were chosen based on the Ministry of Education word frequency database in Taiwan. We first chose 20 of the most frequently used positive/negative words from the database and asked eight additional participants (excluded from the main analyses) to rate the affective level of these words at a 7-point scale (i.e., "how positive/negative is the word?"; range $=-3$ to 3 , negative values represent negative words and vice versa). Five positive and five negative words with highest rating scores were chosen as our experimental materials. These words might also reflect certain positive and negative feelings that a care-receiver may experience when interacting with a caregiver [18], which helps us to understand whether older adults associate such experiences with robots or humans. If participants had stronger association between robots and negative words than humans and negative words, we would expect to observe a faster response when the robot-negative pairs were presented compared to the human-negative pairs. 
The procedure of the IAT we used in the present study (five stages included) was adopted from Nosek et al.'s (2007) work. Stage 1 (20 trials) was the initial target concept discrimination task used to ensure that participants were familiar with the two categories. The silhouettes of robots or humans were presented in a random sequence and categorized by designated keys on the left or right side of the keyboard (e.g., left "E" key for the response of "robot", and right "I" key for the response of "human"). The silhouettes were presented until responses were made. Stage 2 (20 trials) was the associated attribute discrimination task used to practice the categorization of attributes (negative words versus positive words). Participants categorized negative words and positive words by pressing designated keys (e.g., left "E" key for negative words, and right "I" key for positive words). Stage 3 was the first critical phase for compatible combinations. Items representing the concept robot and negative words were categorized with one response and items representing human and positive words were categorized with the alternative response (e.g., left "E" key for humans and positive words, and right "I" key for robots and negative words). Stage 3 consisted of two blocks. Block 1 consisted of only 20 trials because it served as the practice section of Stage 3, but block 2 consisted of 40 trials. Stage 4 was the reversed target discrimination task; responses to target concepts were reversed (e.g., left "E" key for humans, right "I" for robots). Stage 5 was the second critical phase for incompatible combinations. Participants categorized between negative word and human pairings versus positive word and robot pairings (e.g., left "E" key for negative-human pairing and right "I" key for positive-robot pairings). Same as the first critical phase (Stage 3), Stage 5 contained two blocks: 20 trials in block 1 and 40 trials in block 2. And the first block also served as a practice section. The sequence of which pairing was presented and associated with the designated key on the left or right side of the keyboard was counterbalanced across participants.

We also adopted Cohen's $d$ as the index of implicit attitude toward robots, named as the D1 score. Cohen's d was a widely used index of effect size, which was a reference of power in statistical inference. D1 score was calculated by participants' mean reaction time of block 2 at Stage 3 subtracted from that of block 2 at Stage 5, and the difference was further divided by the combined standard deviation of the two critical stages [37].

\subsection{Procedure}

After understanding the procedure of the experiment, all participants gave written informed consent. Participants were required to fill in the basic information form, NARS, TAM, and STAI in the pretest. After the pretest, participants were instructed to interact with the assistive robot based on the 12 commands listed in Table 2. NARS, TAM, and STAI were filled again as the posttest. Participants also completed the questions of state and trait curiosity after interacting with the assistive robots. In the end, participants were instructed to perform the IAT.

\section{RESULTS AND DISCUSSION}

\subsection{NARS}

We performed two-way mixed analysis of variance (ANOVA, within-subject factor: pre-interaction versus post-interaction; between-subject factor: younger versus older adults) on the three subscales of NARS separately. For the FIS subscale (Figure 3(a)), there was a marginally significant effect of interacting experience, $F(1,46)=3.85, p=.056, \eta p^{2}=.077$. The main effect of age was not significant, $F(1,46)=.54, p=.468, \eta p^{2}=.012$. The interaction between age and interacting experience was not significant, either, $F(1,46)=.08, p=.781, \eta \mathrm{p}^{2}=.002$. For the RA subscale (Figure 3(b)), a significant main effect of interacting experience was observed, $F(1,46)=13.51$, $p<$ $.01, \eta \mathrm{p}^{2}=.227$, but not the main effect of age, $F(1,46)=.190, p=.662, \eta \mathrm{p}^{2}=.004$. There was no significant interaction between age and interacting experience, $F(1,46)=.050, p=.825, \eta \mathrm{p}^{2}=.001$. 

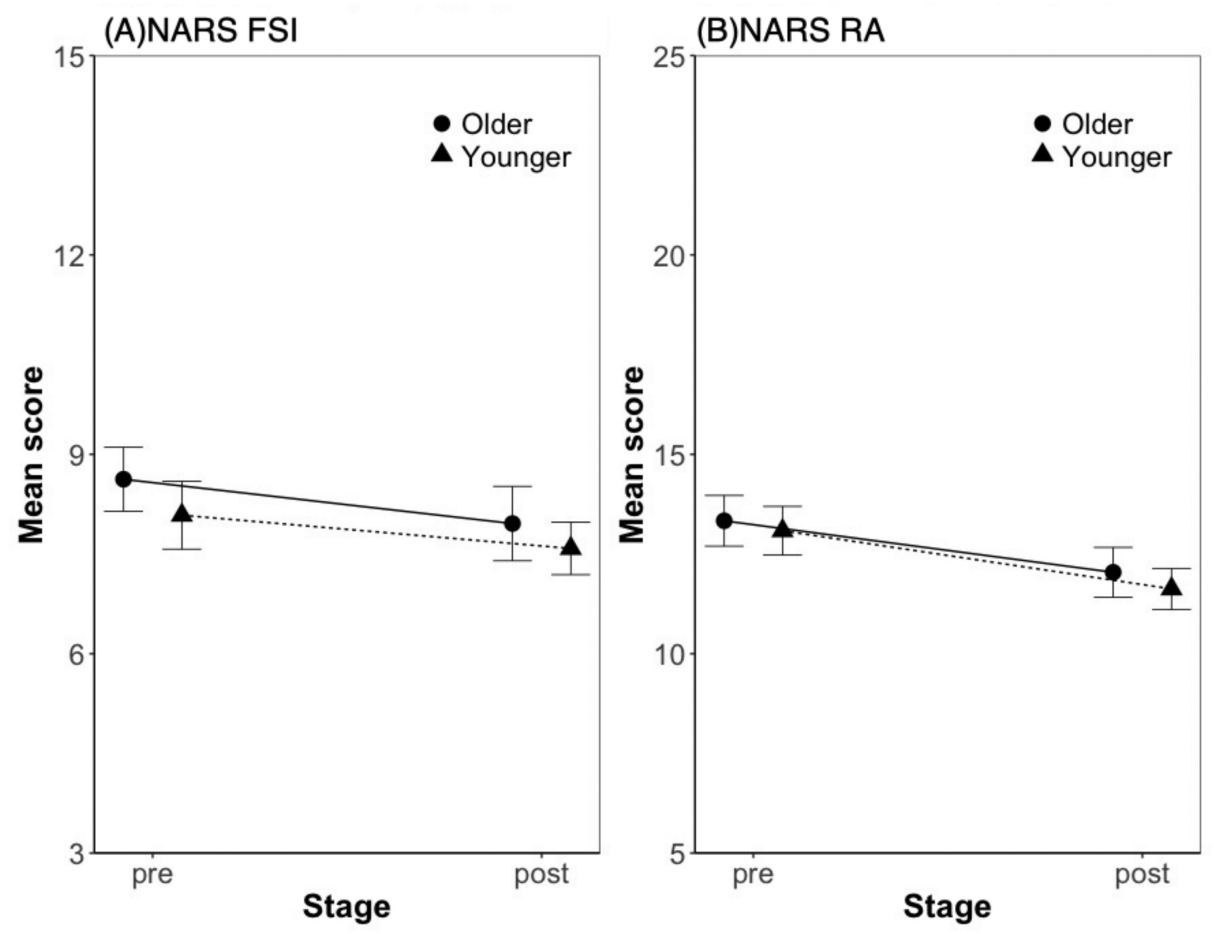

Fig. 3. Results of the (A) NARS future social influence (FSI) and (B) NARS relational attitudes (RA) subscale scores. For both subscales, younger and older participants showed decreased scores after interacting with the assistive robot. Error bars represent one standard error from the mean.

For the AIS subscale, the main effects of both interacting experience $(F(1,46)=.685, p=0.412$, $\left.\eta \mathrm{p}^{2}=.015\right)$ and age $\left(F(1,46)=.027, p=.870, \eta \mathrm{p}^{2}=.001\right)$ were not significant. There was no significant interaction of age and interacting experience, $F(1,46)=.027, p=.869, \eta \mathrm{p}^{2}=.001$.

The RA subscale measured participants' negative attitude toward interacting with robots having emotion (e.g., "I feel comforted being with robots that have emotion.") and the FSI subscale measured participants' negative attitudes for possible future influence of robots (e.g., "I feel that if I depend on robots too much, something bad might happen."). Comparing the two NARS subscales before (FSI: $M=8.35, S D=2.42$; RA: $M=13.2, S D=3.02$ ) and after (FSI: $M=7.77, S D=$ 2.35; RA: $M=11.8, S D=2.78$ ) interacting with the assistive robot, the results showed that negative attitudes toward robots for both younger and older participants decreased after interacting with the assistive robot.

\subsection{TAM}

We performed two-way mixed ANOVAs (within-subject factor: pre-interaction versus postinteraction; between-subject factor: younger versus older) on the three subscales scores of TAM separately as we did for NARS scores. There was a significant main effect of age, $F(1,46)=4.84$, $p<.05, \eta p^{2}=.095$ in the PEU subscale (Figure 4), suggesting that the PEU subscale scores of the older participants were significantly higher than younger participants. This effect implies that older participants' perceived ease of use for the assistive robot was higher than younger participants, both before (older adults: $M=15.0, S D=2.46$; younger adults: $M=14.1, S D=2.13$ ) and after (older adults: $M=15.3, S D=1.88$; younger adults: $M=13.6, S D=2.55$ ) interacting with the 


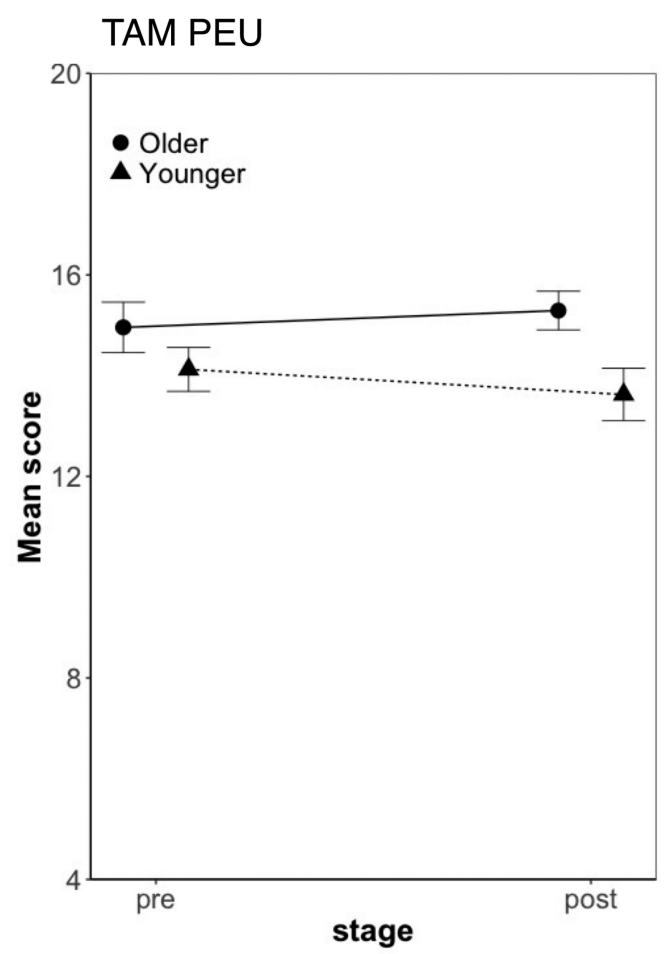

Fig. 4. Results of the perceived ease of use (PEU) subscale of TAM. Older participants showed higher TAMPEU scores than younger participants. Error bars represent one standard error from the mean.

assistive robot. The main effects of interacting experience $\left(F(1,46)=.07, p=.800, \eta p^{2}=.001\right)$, and interaction of age and interacting experience $\left(F(1,46)=1.63, p=.208, \eta \mathrm{p}^{2}=.034\right)$ were not significant. In the PU subscale, there were no significant main effects of both age $(F(1,46)=.172$, $p=$ $\left..681, \eta \mathrm{p}^{2}=.004\right)$ and interacting experience $\left(F(1,46)=.388, p=.537, \eta \mathrm{p}^{2}=.008\right)$, and no significant interaction between age and interacting experience, $F(1,46)=2.531, p=.118, \eta p^{2}=.052$. In the IU subscale, there were no significant main effects of age $\left(F(1,46)=.082, p=.776, \eta \mathrm{p}^{2}=.002\right)$ and interacting experience $\left(F(1,46)=1.686, p=.201, \eta \mathrm{p}^{2}=.036\right)$, and no interaction between age and interacting experience, $F(1,46)=1.128, p=.294, \eta \mathrm{p}^{2}=.024$.

\subsection{STAI}

We performed two-way mixed ANOVAs (within-subject factor: pre-interaction versus postinteraction; between-subject factor: younger versus older) on the three subscales scores of STAI. We found a significant main effect of interacting experience (Figure 5) in the PST subscales, $F(1$, $46)=4.82, p<.05, \eta \mathrm{p}^{2}=.095$. However, both the main effect of age $\left(F(1,46)=1.49, p=.229, \eta \mathrm{p}^{2}=\right.$ $.031)$ and the interaction effect $\left(F(1,46)=.43, p=.517, \eta \mathrm{p}^{2}=.009\right)$ were not significant. We also observed marginally significant effect of interacting experience in the TGE subscale, $F(1,46)=$ 4.03; $p=.0505, \eta \mathrm{p}^{2}=.081$. And the main effect of age $\left(F(1,46)=.43, p=.514, \eta \mathrm{p}^{2}=.009\right)$ and the interaction effect $\left(F(1,46)=.767, p=.386, \eta \mathrm{p}^{2}=.016\right)$ were not significant. Both PST and TGE scores were higher after interacting with robots (before interaction, PST: $M=14.8, S D=2.01$; TGE: $M=15.5, S D=2.84$; after interaction, PST: $M=15.6, S D=2.39$; TGE: $M=16.3, S D=2.11$ ). The findings of increased TGE scores suggest that participants became more willing to learn how to use the assistive robot after interaction, and have stronger feelings that the assistive robot is safe 

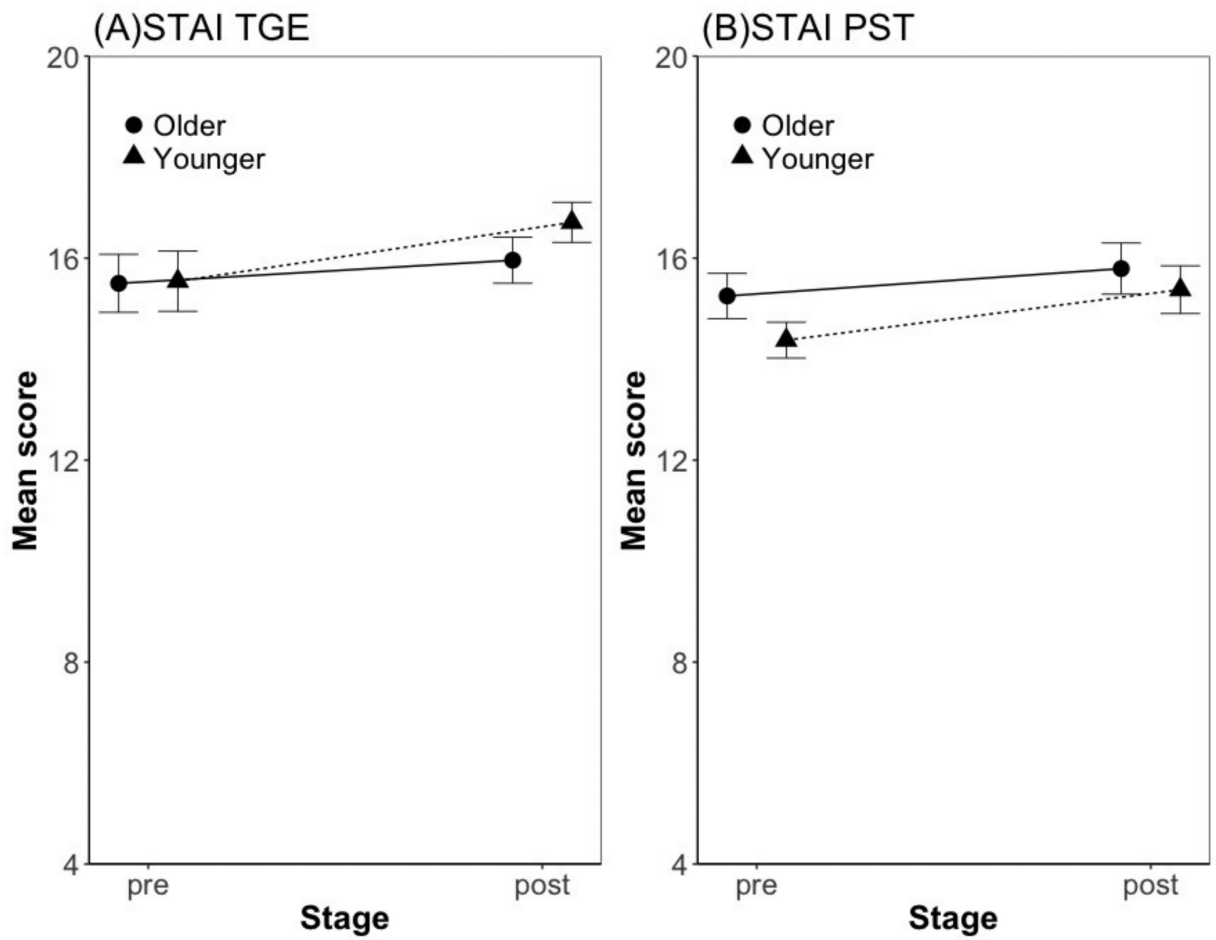

Fig. 5. Results of the technology-related goal-engagement (TGE) and perceived safety of technology (PST) subscale scores of STAI. Participants showed increased scores of the TGE and PST subscales after interacting with the assistive robot. Error bars represent one standard error from the mean.

and can be trusted after interaction. In the PAU subscale, the main effects of age $(F(1,46)=1.52$, $\left.p=0.224, \eta \mathrm{p}^{2}=.032\right)$ and interacting experience $\left(F(1,46)=1.764, p=.193, \eta \mathrm{p}^{2}=.036\right)$ were not significant. The interaction between age and interacting experience was not significant, either, $F(1$, 46) $=.137, p=.713, \eta p^{2}=.003$.

\subsection{State Curiosity toward Robots}

A one-way ANOVA was performed to test the age group difference in state curiosity toward robots. After controlling for the effects of sex, education, and trait curiosity, there was a significant age difference in state curiosity toward robots, $F(1,41)=4.30, p=.04, \eta \mathrm{p}^{2}=.10$. Pairwise comparison showed that older adults' level of state curiosity $(M=15.50 ; S D=3.33)$ was significantly lower than younger adults $(M=16.92 ; S D=3.56$; Figure 6$)$.

We then took personal association into account using multilevel linear regression. After controlling for sex, education, and trait curiosity, excluding one participant with extreme personal association score (score of 1 ), we found a significant overall fit by including age, personal association, and the interaction term, $F(6,38)=7.42, M S E=2.17, p<.001, \mathrm{R}^{2}=.54$. Specifically, the interaction between age groups and personal association was significant, $\beta=.43, S E=.76, p<$ .01 . We conducted a simple slope analysis to examine age difference at different levels of personal association, and did a median split in order to categorize personal association into high (score 5 or above) versus low groups (score 4 or below). The simple slope analysis revealed that, among individuals who scored lower on personal association, there was a significant age group difference, mean difference (Older-Younger) $=-3.60, S E=1.04, p=.001,95 \% \mathrm{CI}=[-5.51,-1.51]$. Yet, 


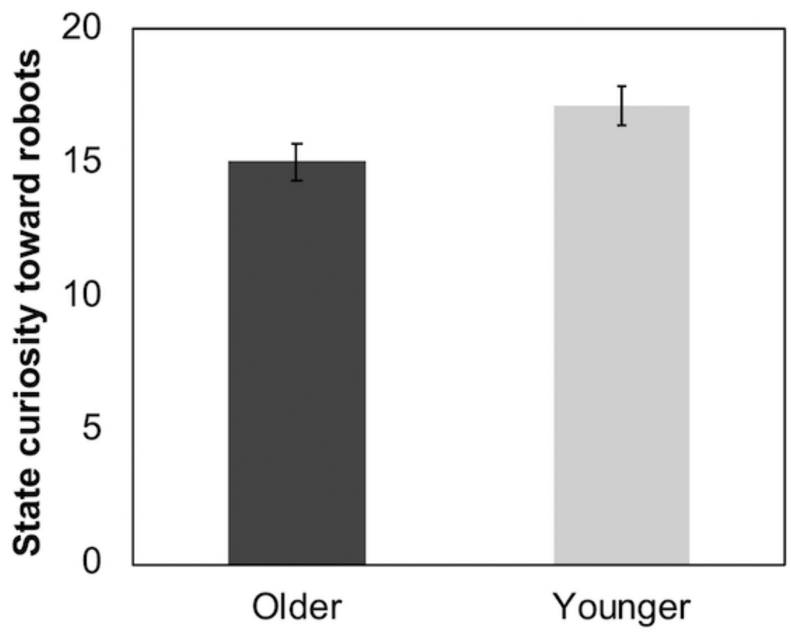

Fig. 6. Age difference in state curiosity scores toward robots. Older participants' score (black bar) was significantly lower than younger participants' score (gray bar). Error bars represent one standard error from the mean.

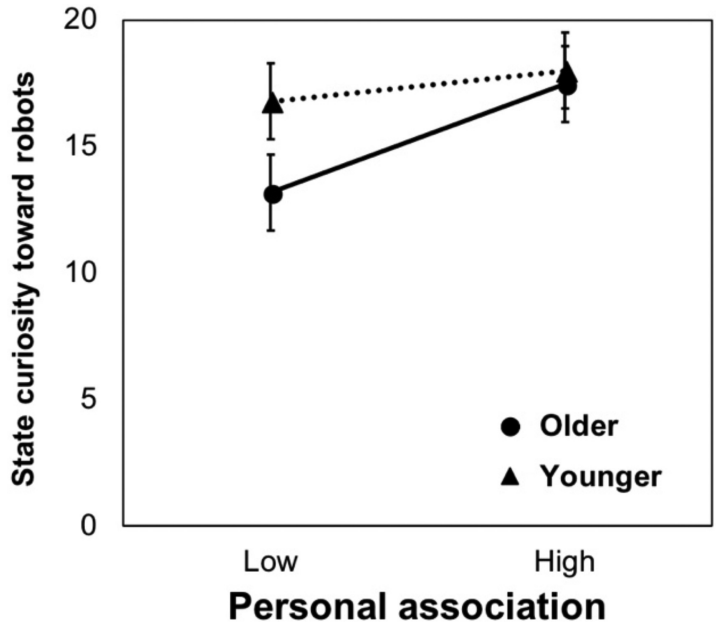

Fig. 7. Age difference in the moderation effect of personal association on state curiosity toward robots. Age group difference was shown only among participants who reported lower personal association. Error bars represent one standard error from the mean.

among individuals who reported a higher personal association, there was no age group difference (Figure 7).

\subsection{IAT}

An independent-samples t-test showed that the average D1 score of the older participants $(M=$ $0.64, S D=0.35)$ was significantly higher than that of younger participants $(M=0.39, S D=0.32$; $t(45.67)=2.55, p<.05$; see Figure 8 ). It suggests that older participants have more strongly associated robots with negative emotions than younger participants. That is, older participants generally have a more negative attitude toward robots than younger participants implicitly. 


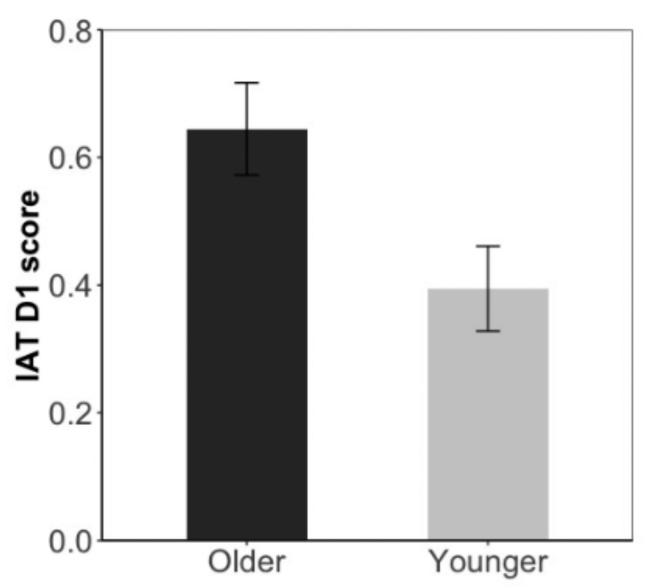

Fig. 8. The D1 scores of IAT. Older participants' D1 score (black bar) was higher than younger participants' (gray bar). Error bars represent one standard error from the mean. Older participants are more strongly associated robots with negative emotions than younger participants.

\section{DISCUSSION}

In the present study, we investigated (1) whether older adults' experience of interacting with robots would affect their explicit attitudes and/or acceptance toward robots compared to younger adults; (2) whether state curiosity toward robots differs between the two age groups; and (3) whether older adults implicitly hold more negative attitudes toward robots than younger adults. To address the first research question, we examined whether there exists an age difference in pretest-posttest explicit attitude ratings. We found age-related differences in TAM-PEU scores. Surprisingly, perceived ease of use toward the assistive robot was stronger in older adults than in younger adults, even after interacting with the robot. This might seem to be contradictory to the IAT results, suggesting that older adults more strongly associated robots with negative attributes. One possible explanation is that younger adults are more experienced with new technologies such as the voice control function. Their standard of "perceived ease of use" might, therefore, be higher than older adults'. For example, if the assistive robot could not respond to voice commands immediately, younger adults might become annoyed due to the long response latency. Then they might think that the usability of the assistive robot was not good. On the other hand, older adults might be less familiar with new technologies. Thus, once they found that they could communicate with the assistive robot with voice commands, even if the tasks were as simple as searching news on the Internet and it might take a while for the assistive robot to complete this task, it was still sufficient for older adults to be satisfied because they did not have to control the robot via the "complicated" graphical user interface. Previous studies suggested that technology experience influenced robot acceptance across age groups [20]. Perceived enjoyment also enhanced robot acceptance in older adults [10]. For older adults with lower technology familiarity, positive experience might be a key factor to the enhancement of robot acceptance. However, we did not observe other age-related differences in other explicit measures of NARS, TAM, and STAI, suggesting that the explicit attitudes and acceptance toward the assistive robot for the older and the younger participants were similar in many other aspects.

Our results showed that both older and younger adults' explicit attitudes became more positive after interacting with the assistive robot. The decreased NARS-FSI scores and the increased 
STAI-PST scores suggested that both younger and older adults were more willing to believe that robots would not have a bad influence on their daily life and could be trusted after interaction with the robot. These changes might result from the increased familiarity with the assistive robot after interacting with it. Such difference might also be related to the mere exposure effect; that is, as people receive repetitive exposure to a stimulus, they tend to develop a preference toward the stimulus simply due to familiarity [38]. It should be noted that the limitation of the mere exposure effect is constrained to stimuli that are initially perceived as pleasant or at least neutral [39], and this, in turn, could also predict lower acceptance toward robots if the experience of interaction was not positive. In this study, we instructed participants to interact with the assistive robot with a pre-decided task list, and almost all individuals in both age groups could successfully complete the listed tasks. The interacting experience should be positive, or at least neutral; therefore, the increased familiarity of robots resulted in a stronger positive attitude toward robots.

NARS, TAM, and STAI are all commonly used models to analyze explicit attitudes toward technologies, but each model has its own distinctive features. TAM is widely used to study acceptance toward various technologies such as robots and computers across different age groups with excellent stability and reliability $[7,8,10-14]$. NARS focuses on investigating negative attitudes, particularly anxieties toward robots [34] and has also been applied to study older adults' attitudes toward robots [17]. STAI is designed to estimate the adaptivity of technology specifically for older adults [32]. In the present study, the age-related difference was found in the TAM-PEU subscale. Perhaps, TAM is more sensitive to probe age-related differences regarding the acceptance toward robots.

For our second research question, we first compared younger and older adults' state curiosity toward robots and found a significant difference: older adults appeared to be less curious about the robot than younger adults. We then examined the role of personal association and found a significant moderating effect. Our findings support our hypothesis that older adults were less curious than younger adults in general; yet, this age difference was moderated by personal association. In other words, for topics that older adults perceived as a higher level of personal meaning and relatedness, they would invest up to the same level of curiosity in the topic as would younger adults. At first glance, this finding on state curiosity may seem to be in conflict with the findings of our other explicit attitudinal measures (i.e., we found age difference in state curiosity but not in other acceptance measures except the TAM PEU subscale). However, the nature of curiosity is slightly different from mere acceptance in the sense that curiosity captures the willingness to invest energy and resources to obtain information. Hence, it may be seen as one step further from simple likes or dislikes, which may explain why the result differs from the other explicit attitudinal measures. According to the selective engagement theory [21], people would become more selective in how they allocate their cognitive resources with age. It could be related to a well-supported fact that the older adults are less willing to learn technology via trail-and-error compared to other training methods such as reading a printed manual [40]. The Diffusion of Innovation theory [41] points out that trialability or testability are keys for potential adopters to evaluate an innovation, and it would predict that older adults are less likely to be early adopters of new technology (e.g., robots) compared to younger adults because older adults might be less willing to actually use it. However, the selection would be based on personal association. However, previous studies did not test this hypothesis in the context of willingness to allocate time and effort for technology-related information seeking. Thus, the present study offers some insights into the usefulness of the selective engagement theory in the context of technology-related curiosity. This part of the study has the limitation of measuring personal association with a single item. Also, this study cannot provide evidence for causation. Future studies should consider including a more comprehensive scale to capture curiosity or directly manipulate one's personal association for a technological product in 
an experiment. Despite these limitations, this finding is particularly interesting because it suggests the role of personal association on curiosity, and this has vast implications for future robotic designs, adult education, and other services to older adults. Echoing the findings of other explicit attitude measures, we recommend future designers of services dedicated for older adults to include some elements of personal association/relevance (i.e., personalized familiarity or relatedness).

To address the third research question, we compared the D1 score between older and younger adults in IAT. We found that older adults more likely associated robots with negative words. The results of the present study are significant in two aspects: (1) MacDorman and colleagues previously found that older adults associated robots with weapons more strongly than younger adults in IAT [25]. Our results further suggest that older adults tended to associate robots with negative concepts to a greater extent than younger adults in IAT, suggesting that older adults showed stronger negative attitudes toward robots than younger adults. Also, MacDorman et al.'s study mainly focused on comparing whether cultural differences would affect attitudes toward robots. Therefore, to the best of our knowledge, the present study was the first one aimed to examine the effect of age on implicit attitudes toward robots. (2) Our results showed an age difference in the discrepancy between implicit and explicit attitudes. While explicit measures suggested that older adults did not show more negative attitudes toward robots than younger adults, implicit measures suggested that older adults associated robots with a negative impression more strongly than younger adults.

At first, this finding seemed to contradict the results of explicit measures using questionnaires, which suggested that older adults did not show stronger negative attitudes than younger adults. The discrepancy might result from implicit and explicit attitudes being shaped by different processes [24]. Rydell and McConnell suggested that explicit attitudes, which adopt a fast-changing process, were affected by explicit goals and predicted more deliberate behavioral intentions. On the other hand, implicit attitudes, which predict spontaneous behaviors, were shaped by repeated pairings between target objects and related evaluations. Furthermore, the process of shaping implicit attitudes could be slower than explicit attitudes. In the present study, the experience of interaction was shown to change explicit attitudes toward robots. However, changing implicit attitudes might require more time. Perhaps, with sufficient counter information, it is possible to change the stronger negative attitudes of the older adults toward robots. In the case of human-robot interaction, direct interaction with real robots could serve as the counter information. That is, if older adults have a more positive experience of interacting with robots, then implicit negative attitudes may also be modified.

\subsection{Limitations and Future Directions}

The present study aimed to examine differences in attitudes toward robots across age groups; younger participants were recruited from the neighborhood of the university campus and were all college students. However, recruiting college students as younger participants also narrowed down the age range of the younger adults to only 4 years (i.e., 20-24 years) while the age range of the older adults was much larger than the younger adults (i.e., 59-86 years). In addition, the younger adults in the present study might only be representative of college students but not others (e.g., kids, teenagers, middle-aged adults) and might reduce the generalizability of the present study. Future research may recruit participants from a broader range of age to examine whether our findings can be applied across various age groups.

Unlike other explicit measures, IAT and state curiosity were only measured in the posttest. In our preliminary study, we tested if implicit attitude toward robots could be changed after interacting with the assistive robot. The results showed that there was no difference between pretest and posttest in IAT. It was in accordance with the slower shaping of implicit attitudes compared to 
explicit attitudes as previous research suggested [24]. Therefore, we decided to ask participants to perform IAT only after interacting with the assistive robot to simplify the procedures in the present study. However, since state curiosity was measured as a momentary form of explicit attitude, it is hard to clarify whether the age difference in state curiosity was affected by the interacting experience or not. Future research is warranted to investigate whether there is an interaction effect between age difference in state curiosity and interacting experience.

Another limitation of the IAT paradigm used in the present study is that it compared relative strengths of pairs of associations (e.g., negative-robot vs. negative-human pairings) by measuring the speed of response. It could not measure the strength of a single association (e.g., negativerobot). It would be interesting to apply modified IAT paradigms such as the Single Category Implicit Association Test (SC-IAT) [42] to measure the strength of a single association between different age groups. Moreover, the speed of response could be affected by various factors and thus we adopted IAT D1 score as the index of effect size rather than directly comparing the response latency between two age groups in the present study. Because IAT has been shown to be a reliable measure for implicit attitude [43], especially when individuals are unaware of the sources of influence on their behaviors [44], we used IAT in the current study to measure implicit negative impression toward robots in general and adopted silhouettes as stimuli based on MacDorman et al.'s study [25]. However, appearance of robots in the IAT might have influences on participants' implicit attitudes. Future studies may try to modify the IAT paradigm to test this possibility.

In addition, we examined the effect of interaction experience on explicit and implicit attitudes in a relatively short period of time; thereby, it was hard to examine changes in implicit attitudes with the cross-sectional design used in this study. Future researches with a longitudinal design could help clarify whether sufficient interacting experience (e.g., using a robot for a longer period of time) could actually change implicit attitudes.

Finally, we provided a task list for adults to interact with a robot, but actual interactions with robots are likely to be less standardized and more variable. Future research should focus on how to bring positive experience into human-robot interaction when people interact with social robots such as the assistive one we used here.

\section{CONCLUSIONS}

Our results showed that implicit attitudes toward robots were more negative in older adults than in younger adults. Nevertheless, older adults' explicit attitudes toward robots could become more positive as could younger adults if (1) they could successfully interact with the robot and (2) they felt more personal relevance to the robot. Previous studies have pointed out that the key component of user intention is users' attitude toward the product, and the intention to use is a prerequisite for eventual product adoption/rejection [3,35]. Hence, positive attitudes toward robots could be helpful for enhancing the acceptance of robots. The present study suggests that personally relevant positive interactive experiences are the key to enhancing human-robot interaction, especially for older adults. To introduce robots within the care home setting to provide assistance for older adults, understanding how to enhance older adults' acceptance of robots is very important $[2,9-14,18]$. First, when designing home assistive robots, designers should consider including elements of personal relevance, for example, allowing customized designs and settings; thereby, users could build customized robots highly relevant to themselves. Second, since the interacting experience is one of the key factors to enhance the acceptance toward robots, policymakers should consider emplacing robots in public areas for demonstrations to allow the general public to have more opportunity to interact with robots. As implicit attitudes could be shaped by accumulated experience, older adults' stronger negative implicit attitudes toward robots could be reduced via increasing opportunities for older adults to interact with robots. 


\section{ACKNOWLEDGMENTS}

This research was supported by Grants from Taiwan's Ministry of Science and Technology to SY (MOST 107-2410-H-002 -129 -MY3; 106-2420-H-002 -010 -MY3; 107-2634-F-002 -018 ), and SC (MOST 107-2811-H-002-536 and 106-2811-H-002-015). We also thank Dr. Lewis Liu for providing this opportunity for us to collaborate with ITRI.

\section{REFERENCES}

[1] Yi-Yin Lin and Chin-Shan Huang. 2015. Aging in Taiwan: Building a society for active aging and aging in place. The Gerontologist 56, 2 (2015), 176-183.

[2] Marcel Heerink, Ben Kröse, Bob Wielinga, and Vanessa Evers. 2006. Studying the acceptance of a robotic agent by elderly users. Int. F. Assistive Rob. Mechatron. 7, 3 (2006) 33-43.

[3] Yvonne Barnard, Mike D. Bradley, Frances Hodgson, and Ashley D. Lloyd. 2013. Learning to use new technologies by older adults: Perceived difficulties, experimentation behaviour and usability. Comput. Hum. Behav. 29, 4 (2013), 1715-1724.

[4] Anthony G. Greenwald and Mahzarin R. Banaji. 1995. Implicit social cognition: Attitudes, self-esteem, and stereotypes. Psychol. Rev. 102, 1 (1995), 4-27.

[5] Brian A. Nosek. 2007. Implicit-explicit relations. Curr. Directions Psychol. Sci. 16, 2 (2007), 65-69.

[6] John F. Dovidio, Kerry Kawakami, and Samuel L. Gaertner. 2002. Implicit and explicit prejudice and interracial interaction. f. Personality Social Psychol. 82, 1 (2002), 62-68.

[7] Marcel Heerink, Ben Kröse, Vanessa Evers, and Bob Wielinga. 2010. Assessing acceptance of assistive social agent technology by older adults: The Almere model. Int. f. Social Rob. 2, 4 (2010), 361-375.

[8] Hee-dong Yang and Youngjin Yoo. 2004. It's all about attitude: Revisiting the technology acceptance model. Decis. Support Syst. 38, 1 (2004), 19-31.

[9] Rebecca Q. Stafford, Bruce A. MacDonald, Chandimal Jayawardena, Daniel M. Wegner, and Elizabeth Broadbent. 2014. Does the robot have a mind? Mind perception and attitudes towards robots predict use of an eldercare robot. Int. F. Social Rob. 6, 1 (2014), 17-32.

[10] Marcel Heerink, Ben Kröse, Bob Wielinga, and Vanessa Evers. Enjoyment intention to use and actual use of a conversational robot by elderly people. In Proceedings of the 3rd ACM/IEEE International Conference on Human Robot Interaction. ACM, 113-120.

[11] Jenay M. Beer, Akanksha Prakash, Cory-Ann Smarr, Tiffany L. Chen, Kelsey Hawkins, Hai Nguyen, Travis Deyle, Tracy L. Mitzner, Charles C. Kemp, and Wendy A. Rogers. 2017. Older users' acceptance of an assistive robot: Attitudinal changes following brief exposure. Gerontechnology 16, 1 (2017), 21-36.

[12] Tiffany L. Chen, Tapomayukh Bhattacharjee, Jenay M. Beer, Lena H. Ting, Madeleine E. Hackney, Wendy A. Rogers, and Charles C. Kemp. 2017. Older adults' acceptance of a robot for partner dance-based exercise. PLOS ONE 12, 10 (2017), e0182736.

[13] I-Han Kuo, Joel M. Rabindran, Elizabeth Broadbent, Yong-In Lee, Ngaire Kerse, Rebecca Stafford, and Bruce A. MacDonald. 2009. Age and gender factors in user acceptance of healthcare robots. In Robot and Human Interactive Communication (ROMAN'09). IEEE, 214-219.

[14] Marcel Heerink. 2011. Exploring the influence of age, gender, education and computer experience on robot acceptance by older adults. In Proceedings of the 6th International Conference on Human-Robot Interaction. ACM, 147-148.

[15] Christoph Bartneck, Tomohiro Suzuki, Takayuki Kanda, and Tatsuya Nomura. 2007. The influence of people's culture and prior experiences with Aibo on their attitude towards robots. AI Soc. 21, 1-2 (2007), 217-230.

[16] Kerstin Dautenhahn, Sarah Woods, Christina Kaouri, Michael L. Walters, Kheng Lee Koay, and Iain Werry. 2005. What is a robot companion-friend, assistant or butler? In Intelligent Robots and Systems (IROS 2005). IEEE, 1192-1197.

[17] Tatsuya Nomura, Takugo Tasaki, Takayuki Kanda, Masahiro Shiomi, Hiroshi Ishiguro, and Norihiro Hagita. 2007. Questionnaire-based social research on opinions of Japanese visitors for communication robots at an exhibition. AI Soc. 21, 1-2 (2007), 167-183.

[18] Massimiliano Scopelliti, Maria Vittoria Giuliani, and Ferdinando Fornara. 2005. Robots in a domestic setting: A psychological approach. Universal Access Inf. Soc. 4, 2 (2005), 146-155.

[19] Takanori Shibata, Kazuyoshi Wada, and Kazuo Tanie. 2004. Subjective evaluation of seal robot in Brunei. In Robot and Human Interactive Communication (ROMAN'04). IEEE, 135-140.

[20] Neta Ezer, Arthur D. Fisk, and Wendy A. Rogers. 2009. Attitudinal and intentional acceptance of domestic robots by younger and older adults. In International Conference on Universal Access in Human-Computer Interaction. Springer, $39-48$. 
[21] Thomas M. Hess, Cassandra M. Germain, Elizabeth L. Swaim, and Nicole L. Osowski. 2009. Aging and selective engagement: The moderating impact of motivation on older adults' resource utilization. f. Gerontology Ser. B: Psychol. Sci. Social Sci. 64, 4 (2009), 447-456.

[22] Todd B. Kashdan, Paul Rose, and Frank D. Fincham. 2004. Curiosity and exploration: Facilitating positive subjective experiences and personal growth opportunities. F. Personality Assess. 82, 3 (2004), 291-305.

[23] Cory-Ann Smarr, Tracy L. Mitzner, Jenay M. Beer, Akanksha Prakash, Tiffany L. Chen, Charles C. Kemp, and Wendy A. Rogers. 2014. Domestic robots for older adults: Attitudes, preferences, and potential. Int. F. Social Rob. 6, 2 (2014), 229-247.

[24] Robert J. Rydell and Allen R. McConnell. 2006. Understanding implicit and explicit attitude change: A systems of reasoning analysis. F. Personality Social Psychol. 91, 6 (2006), 995-1008.

[25] Karl F. MacDorman, Sandosh K. Vasudevan, and Chin-Chang Ho. 2009. Does Japan really have robot mania? Comparing attitudes by implicit and explicit measures. AI Soc. 23, 4 (2009), 485-510.

[26] Leonard M. Giambra, Cameron J. Camp, and Alicia Grodsky. 1992. Curiosity and stimulation seeking across the adult life span: Cross-sectional and 6-to 8-year longitudinal findings. Psychol. Aging 7, 1 (1992), 150-157.

[27] Stacy J. Priniski, Cameron A. Hecht, and Judith M. Harackiewicz. 2018. Making learning personally meaningful: A new framework for relevance research. f. Exp. Educ. 86, 1 (2018), 11-29.

[28] United Nations, Department of Economic and Social Affairs, Population Division. 2009. World Population Prospects: The 2008 Revision, Highlights, Working Paper No. ESA/P/WP.210.

[29] Tatsuya Nomura, Tomohiro Suzuki, Takayuki Kanda, and Kensuke Kato. 2006. Altered attitudes of people toward robots: Investigation through the negative attitudes toward Robots scale. In Proc. AAAI-06 Workshop on Human Implications of Human-Robot Interaction. 29-35.

[30] Fred D. Davis. 1989. Perceived usefulness, perceived ease of use, and user acceptance of information technology. MIS Quarterly 13, 3 (1989), 319-340. DOI : 10.2307/249008

[31] Fred D. Davis, Richard P. Bagozzi, and Paul R. Warshaw. 1989. User acceptance of computer technology: A comparison of two theoretical models. Manage. Sci. 35, 8 (1989), 982-1003.

[32] Stefan T. Kamin and Frieder R. Lang. 2013. The subjective technology adaptivity inventory (STAI): A motivational measure of technology usage in old age. Gerontechnology 12, 1 (2013), 16-25.

[33] Seong-Hee Park, Daniel F. Mahony, Yukyoum Kim, and Young Do Kim. 2015. Curiosity generating advertisements and their impact on sport consumer behavior. Sport Manage. Rev. 18, 3 (2015), 359-369.

[34] Dag Sverre Syrdal, Kerstin Dautenhahn, Kheng Lee Koay, and Michael L. Walters. 2009. The negative attitudes towards robots scale and reactions to robot behavior in a live human-robot interaction study. In Adaptive and Emergent Behaviour and Complex Systems: Proc. of the 23rd Convention of the Society for the Study of Artificial Intelligence and Simulation of Behaviour (AIS '09). SSAISB, 109-115.

[35] Viswanath Venkatesh and Fred D. Davis. 2000. A theoretical extension of the technology acceptance model: Four longitudinal field studies. Manage. Sci. 46, 2 (2000), 186-204.

[36] Anthony G. Greenwald, Debbie E. McGhee, and Jordan L. K. Schwartz. 1998. Measuring individual differences in implicit cognition: The implicit association test. F. Personality Social Psychol. 74, 6 (1998), 1464-1480.

[37] Klaske A. Glashouwer, Fren T. Y. Smulders, Peter J. de Jong, Anne Roefs, and Reinout W. H. J. Wiers. 2013. Measuring automatic associations: Validation of algorithms for the Implicit Association Test (IAT) in a laboratory setting. $\mathcal{F}$ Behav. Ther. Exp. Psychiatry 44, 1 (2013), 105-113.

[38] Robert B. Zajonc. 1968. Attitudinal effects of mere exposure. F. Personality Social Psychol. 9, 2 (1968), 1-27.

[39] Çağri Imamoğlu and E. Olcay Imamoğlu. 2006. Relationship between familiarity, attitudes and preferences: Assisted living facilities as compared to nursing homes. Social Indic. Res. 79, 2 (2006), 235-254.

[40] Tracy L. Mitzner, Cara B. Fausset, Julie B. Boron, Anne E. Adams, Katinka Dijkstra, Chin Chin Lee, Wendy A. Rogers, and Arthur D. Fisk. 2008. Older adults' training preferences for learning to use technology. In Proceedings of the Human Factors and Ergonomics Society Annual Meeting, Vol. 56(26). SAGE Publications, 2047-2051.

[41] Everett M. Rogers. 2003. Diffusion of Innovations (5th Edition). Free Press, New York, NY.

[42] Andrew Karpinski and Ross B. Steinman. 2006. The single category implicit association test as a measure of implicit social cognition. f. Personality Social Psychol. 91, 1 (2006), 16-32.

[43] William A. Cunningham, Kristopher J. Preacher, and Mahzarin R. Banaji. 2001. Implicit attitude measures: Consistency, stability, and convergent validity. Psychol. Sci. 12, 2 (2001), 163-170.

[44] Frederic F. Brunel, Brian C. Tietje, and Anthony G. Greenwald. 2004. Is the implicit association test a valid and valuable measure of implicit consumer social cognition? f. Consum. Psychol. 14, 4 (2004), 385-404.

Received August 2018; revised January 2019; accepted January 2019 